\title{
Desmocollin-3: a new marker of squamous differentiation in undifferentiated large-cell carcinoma of the lung
}

\author{
Valentina Monica ${ }^{1}$, Paolo Ceppi ${ }^{1}$, Luisella Righi ${ }^{1}$, Veronica Tavaglione ${ }^{1}$, Marco Volante ${ }^{1}$, \\ Giuseppe Pelosi ${ }^{2}$, Giorgio V Scagliotti ${ }^{1}$ and Mauro Papotti ${ }^{1}$ \\ ${ }^{1}$ Department of Clinical and Biological Sciences, San Luigi Hospital, University of Turin, Torino, Italy \\ and ${ }^{2}$ Division of Pathology, European Institute of Oncology and University of Milan School of Medicine, \\ Milan, Italy
}

\begin{abstract}
Lung cancer classification in small-cell and non-small-cell types was recently challenged by data on the differential efficacy of new cytotoxic agents in specific histotypes. An accurate histotype definition has therefore gained interest in both preoperative and surgical materials, but is a hard task especially in undifferentiated large-cell tumors lacking morphological signs of squamous or glandular differentiation. The responsiveness of these latter subtypes to new drugs apparently more selective for adenocarcinomas or squamous carcinomas is not fully understood, also due to the heterogeneity of diagnostic criteria for this tumor entity. Current immunohistochemical markers are not fully specific and new molecules are to be explored. On the basis of gene expression profiling data, reporting a remarkable differential expression of desmocollin-3 (a protein localized in desmosomal junctions of stratified epithelial) between adeno- and squamous cancers, we immunostained 62 cases of resected undifferentiated large-cell lung carcinomas for desmocollin-3 (and for TTF-1, p63 and mucin stain), to test its ability to identify a (residual) squamous phenotype, if present. Desmocollin-3 was expressed in almost half of the undifferentiated large-cell cancers and was mutually exclusive with TTF-1 (positive in 39\%; the remaining $18 \%$ of cases was double negative). Special large-cell carcinoma variants expressed desmocollin-3 in 6 of 6 basaloid, 7 of 12 clear-cell types, again mutually exclusive with TTF-1 expression. None of seven sarcomatoid carcinomas reacted for either marker. In 31 cytological samples diagnosed as 'non-small-cell lung carcinoma', desmocollin-3 was again mutually exclusive with TTF-1 and stained all squamous carcinomas, 1 of 19 adenocarcinoma only, and $50 \%$ of large-cell carcinoma (all histologically confirmed). This combined morphophenotypic approach may represent a valid adjunct (for both surgical and cytological samples) in the selection of patients with lung cancer to medical treatments tailored according to different efficacy in different lung carcinomas of the squamous, adeno- and large-cell types.
\end{abstract}

Modern Pathology (2009) 22, 709-717; doi:10.1038/modpathol.2009.30; published online 13 March 2009

Keywords: large-cell carcinoma; squamous differentiation; desmocollin-3; diagnosis

Lung cancer is subdivided into four histotypes, including small-cell carcinoma (SCLC) on the one side, and carcinomas of squamous-, adeno- and large-cell types on the other. These latter tumor groups have different pathological features, but have generally been treated so far according to similar strategies, so that a clear-cut distinction among

Correspondence: Professor M Papotti, MD, Department of Clinical and Biological Sciences, Division of Pathology, University of Turin at San Luigi Hospital, Regione Gonzole 10, 10043 Orbassano, Torino, Italy.

E-mail: mauro.papotti@unito.it

Received 07 October 2008; revised and accepted 19 November 2008; published online 13 March 2009 these types (except for SCLC) was not considered clinically relevant. ${ }^{1}$

Indeed, the WHO classifications of lung carcinoma have always kept the different histological types separate and proposed individual categories for squamous carcinoma, adenocarcinoma and large-cell (anaplastic) carcinoma (LCC). ${ }^{2}$ The latter category underwent major changes in the last classification scheme, ${ }^{3}$ having undifferentiated pleomorphic and/or sarcomatoid variants been moved to a new group of 'sarcomatoid carcinoma'. Rare lung cancer variants are incorporated under the heading of LCC, such as lymphoepithelial, clear-cell, rhabdoid, basaloid and the large-cell neuroendocrine carcinoma. Indeed, in the common practice, cases lacking signs of glandular or 
squamous differentiation are reported as 'large cell (anaplastic) carcinoma' (ICD-O code 8012/3), also to exclude the diagnosis of small-cell lung cancer. This is particularly critical when analyzing cytological material or small biopsies, often resulting in a generic diagnosis of 'non-small-cell lung carcinoma', due to the limited amount of viable tissue or cells.

In recent years, the attitude of grouping lung cancers of the non-small-cell types under a single label was challenged, because the efficacy of new drugs, alone or in combination, was found to be correlated to single histotypes of squamous-, adenoand large-cell carcinomas. ${ }^{4-6}$ The recognition that some chemotherapeutic regimes were more effective in squamous-cell carcinomas, whereas other drug combinations were apparently better active in nonsquamous histological types (eg antifolate drugs), brought up the issue of accurately defining the histotypes in both preoperative and surgical materials. The responsiveness of large-cell lung cancers to new drugs apparently more selective for adenocarcinomas or squamous carcinomas is not fully understood, possibly due to the heterogeneous tumor types entered in this group along the years and by different pathologists.

Well-differentiated lung cancer types can be easily characterized by means of histochemical (PAS stain and PAS after diastase for mucins) and immunohistochemical methods (TTF-1 and surfactant for adenocarcinomas, as opposed to p63 and cytokeratins 5 or 17 for squamous carcinomas). ${ }^{7}$ Indeed, these markers are relatively sensitive, but not completely specific. A minor fraction of squamous carcinoma is reported to react with TTF- $1,{ }^{8-10}$ although it is not clear whether these cases are pure squamous or rather combined adenosquamous cancers. On the other side, p63 is considered a marker of squamous epithelia, although recent reports indicate that this protein is also expressed in basal cells of glandular tumors. ${ }^{11}$ Such diagnostic markers are much less reliable in poorly differentiated carcinoma types, although in one study, up to $60 \%$ of LCCs were further subclassified with a panel of the above markers. ${ }^{12}$

Major advances in the phenotypic profiling of different lung cancer subtypes were obtained by molecular analysis of a large number of genes, which seemed to provide different signatures in different histological types, according to their up- or downregulation. ${ }^{13-15}$ In a recent study on non-smallcell lung carcinoma, ${ }^{16}$ four genes were found upregulated in squamous carcinomas by at least 20-fold compared to adenocarcinomas and/or normal lung parenchyma (PKP1, DSC3, p63 and CK17). Among these, the highest difference between squamous carcinoma and adenocarcinoma (and also between cancer and normal tissue) was observed for DSC3, which codes for desmocollin-3, a constitutive protein of desmosomes. In cooperation with desmogleins (adhesion proteins belonging to cadherin family), desmocollins interact through their extracellular domains to hold the adjacent plasma membranes together. Desmoglein-3 was found to be restricted to squamous histology and to bear prognostic significance in lung carcinomas (SCLC excluded). ${ }^{17}$ Conversely, desmocollin-3 has been poorly investigated in the lung. Basal layers of stratified epithelia strongly express desmocollin3 , whereas basal cells of columnar epithelia (eg bronchi and trachea) are occasionally immunoreactive. ${ }^{16,18}$

On the basis of the reported remarkable differential expression between adeno- and squamous cancers, we hypothesized that desmocollin-3 could be a reliable marker to trace (residual) squamous features in undifferentiated large-cell lung carcinomas (and take them apart from adenocarcinomalinked cases). To this purpose, two series of resected LCC and a set of cytological samples diagnosed as non-small-cell lung carcinomas (and their corresponding histological material) were immunostained for desmocollin-3 (in parallel with TTF-1, p63 and mucin stain). This retrospective study shows that desmocollin-3 is expressed in a fraction of undifferentiated LCCs, which is generally mutually exclusive with TTF-1; co-expression of the two markers is restricted to adenosquamous carcinomas. Desmocollin-3 may represent a valid adjunct in the selection of patients with lung cancer to systemic treatments, tailored according to the specific histotype.

\section{Materials and methods}

\section{Surgical Specimens}

Out of a database of approximately 800 completely resected lung carcinomas at the San Luigi Hospital and University of Turin in the period of time 2000-2007, 34 cases were selected, having an original diagnosis of 'anaplastic LCC', 'LCC', 'sarcomatoid carcinoma' or 'undifferentiated carcinoma of the lung'. Clinicopathological data were collected, including sex, age, tumor size and location, and tumor stage. Upon revision of the original hematoxylin and eosin-stained slides, five cases were excluded because they were reclassified as poorly differentiated squamous carcinomas (four cases) or adenocarcinoma (one case), based on the identification of a minor, but unequivocal component of keratinized or glandular type. Of the remaining 29 cases, a representative paraffin-embedded tissue block was selected and serial sections were obtained for histochemical and immunohistochemical stainings. These cases included seven clear-cell variants and three basaloid variants of LCC. Three tumors had a spindle-cell component and represented sarcomatoid carcinomas. The remaining 16 cases were undifferentiated carcinomas, lacking morphological and histochemical (mucin stain) signs of squamous or glandular differentiation, diagnosed as LCC, not otherwise specified (ICD-O code 8012/3). 
An additional series of 33 undifferentiated LCC specimens, completely resected in the same period of time as above (2000-2007) at the European Institute of Oncology in Milan, was included in the present study as a validation cohort, to confirm our results on a different tumor series. Such LCCs encompassed three basaloid, five clear-cell and one lymphoepithelioma variants. Four cases were classified as sarcomatoid carcinomas. The remaining 20 cases were undifferentiated LCCs not otherwise specified (ICD-O code 8012/3).

\section{Control Cases}

A series of well-differentiated squamous-cell carcinomas (20 cases, 4 of which keratinized), invasive adenocarcinomas (20 cases, belonging to acinar, papillary or mixed subtypes) and adenosquamous carcinomas (3 cases) completely resected in the same period at the University of Turin at St Luigi Hospital was collected as a control group, and submitted to the same panel of histochemical and immunohistochemical markers.

All histological material was deidentified and cases were anonymized by a pathology staff member not involved in the study. Clinical data were compared and analyzed through coded data only. The study was approved by the institutional review board of the hospital.

\section{Cytological Material}

A pilot series of 31 cytological samples (16 cases of transthoracic fine-needle aspirates and 15 cases of bronchoalveolar lavage), diagnosed as 'undifferentiated non-small-cell lung carcinoma' not otherwise specified, was also investigated for TTF-1, p63 and desmocollin-3 immunoreactivity in paraffinembedded cell blocks. These cases included 13 advanced-stage lung cancers for which tissue from a bronchial biopsy was also available (it was assumed that the cell population encountered in the biopsy was representative of the whole tumor), whereas the remaining 18 cases were subsequently surgically resected. Representative blocks from either biopsy or surgical tumor tissues were stained in parallel with the corresponding cytological specimens.

\section{Histochemistry and Immunohistochemistry}

From each paraffin block, 5-mm-thick sections were prepared and stained with hematoxylin and eosin for conventional histological examination and with periodic acid-Schiff method, with or without prior treatment with diastase to highlight intracellular glycogen or mucins. In addition, serial sections collected on charged slides were analyzed by immunohistochemistry staining as follows: after paraffin removal and rehydration through graded alcohols and phosphate-buffered saline ( $\mathrm{pH}$ 7.5), endogenous peroxidase activity was blocked by absolute methanol and $0.3 \%$ hydrogen peroxide for $15 \mathrm{~min}$. The slides were incubated for $40 \mathrm{~min}$ at room temperature with primary mouse anti-TTF-1 antibody (dilution 1:75; Neomarkers Thermo Scientific, Fremont, CA, USA) and with primary mouse anti-p63 antibody (dilution 1:100; Neomarkers Thermo Scientific), whereas desmocollin-3 incubation was performed overnight at $4^{\circ} \mathrm{C}$ ( dilution 1:30; Progen Biotechnik, Heidelberg, Germany). The immune reaction was revealed by a biotin-free detection system based on a dextran chain linked to the secondary antibody and carrying peroxidase molecules (EnVision mouse; Dako, Glostrup, Denmark), using 3,3-diaminobenzidine (Dako) as the chromogen. For antigen retrieval, the sections were treated in a pressure cooker for $5 \mathrm{~min}$ at $125^{\circ} \mathrm{C}$ followed by a quick $10 \mathrm{~s}$ step at $90^{\circ} \mathrm{C}$ using EDTA buffer (pH 8.0) for TTF-1 and p63, or citrate buffer for desmocollin3. Slides were counterstained with hematoxylin, dehydrated and mounted. The immune reaction was scored as negative (totally negative, score 0) or positive (presence of an immunohistochemical signal even in single cell membranes or nuclei, for desmocollin-3, or p63 and TTF-1, respectively).

\section{Results}

\section{Surgical Specimens}

In the original series of 29 resected undifferentiated LCC, PAS stain (with or without diastase treatment) revealed focal positivity in 15 cases. p63 was expressed in the majority of cases (26 of 29, 90\%), with no preferential association to either PAS stains nor desmocollin-3 or TTF-1. These latter markers were reactive in 14 and 12 cases, respectively, generally with a mutually exclusive pattern (Figure $1 \mathrm{~d}-\mathrm{o}$ ). The three remaining cases (all sarcomatoid carcinomas, spindle-cell variant) were negative for both markers (Figure 1p-r). Regarding specific histological subtypes or variants, basaloid variants of LCC were TTF-1 negative, whereas desmocollin-3 was expressed in all three such cases (Figure 1d). The seven clear-cell carcinoma variants had a mutually exclusive expression of such markers, being TTF-1 present in four tumors and desmocollin-3 in the other three (Figure 1g-l). In the remaining 16 undifferentiated LCCs (not otherwise specified), a mutually exclusive immunoreactivity for TTF-1 (8 of 16 cases) and desmocollin-3 (8 of 16 cases) in a more or less extensive tumor cell percentage was observed. The immunostaining results for desmocollin-3 and TTF- 1 are summarized in Table 1.

In the validation cohort of 33 undifferentiated LCCs collected at the European Institute of Oncology in Milan, a strong correlation with the above results was demonstrated: desmocollin-3 was 
H\&E
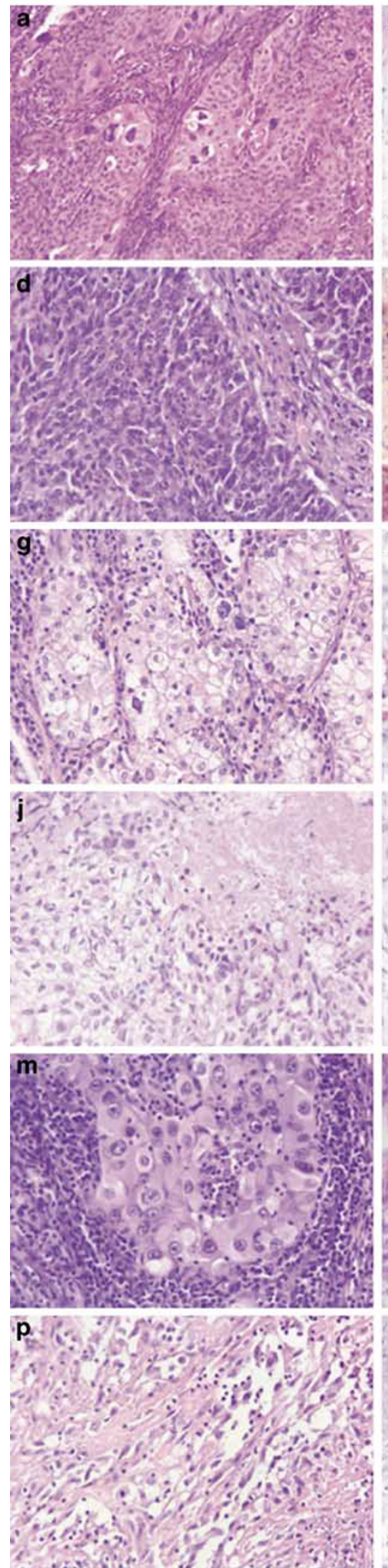

DSC-3
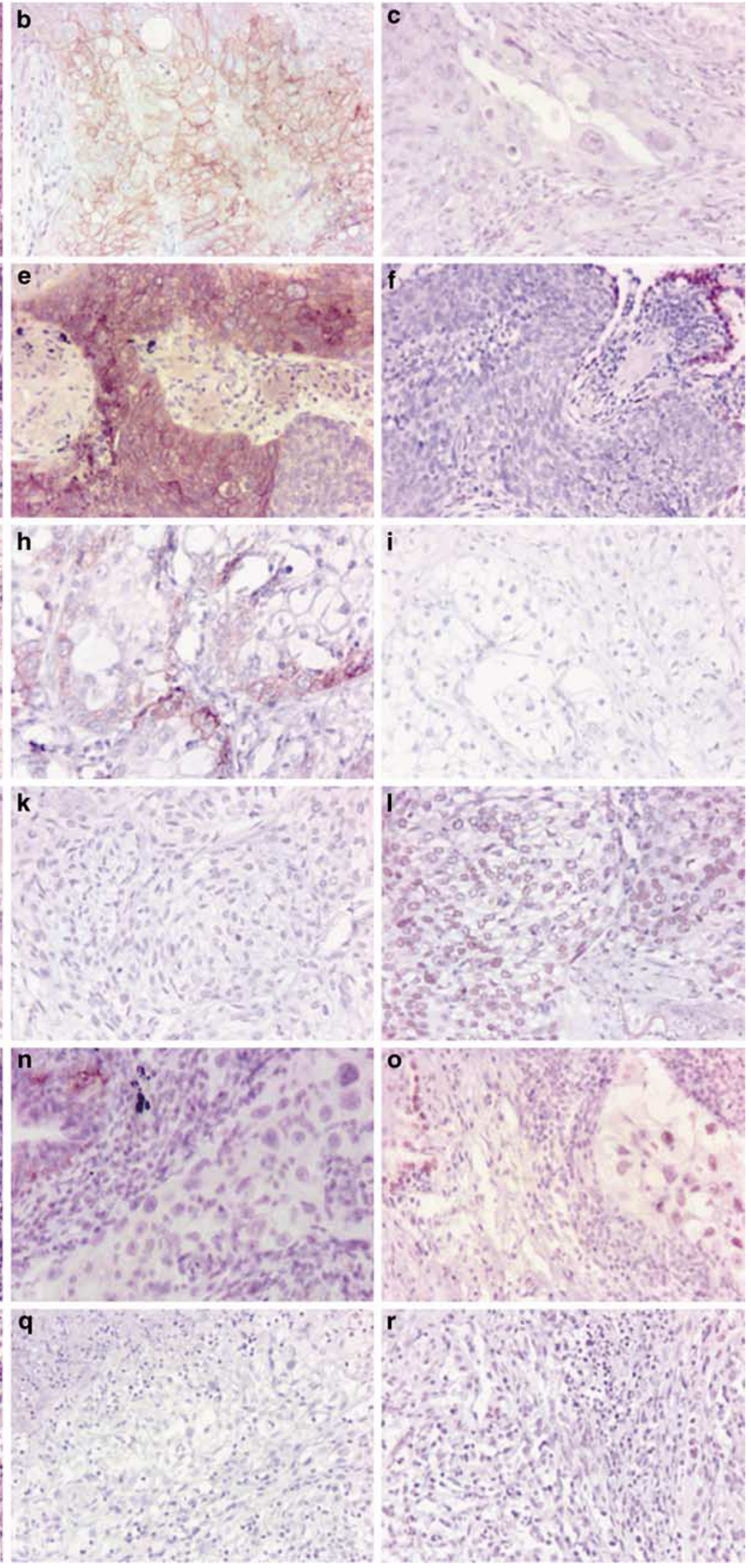
expressed in 13 and TTF-1 in 12 tumors, with a mutually exclusive pattern. All three cases with basaloid features showed an exclusive desmocollin3 staining, as well as the single lymphoepithelioma variant. The five clear-cell carcinoma subtypes expressed selectively desmocollin-3 (four cases) or TTF-1 (the remaining one). The 20 undifferentiated carcinomas not otherwise specified showed mutually exclusive immunoreactivity for both markers, being TTF-1 preferentially expressed (11 of the 20 cases) compared to desmocollin-3, detected in 5 other tumors. The remaining 4 of 20 LCC cases were negative for both markers. Among the 11 TTF-1positive cases, 4 had neuroendocrine morphology, according to nuclear and structural features: although no neuroendocrine markers were expressed in this subgroup of tumors, it cannot be excluded that the TTF-1 reactivity is related to the known strong TTF-1 expression by poorly differentiated neuroendocrine small-cell carcinomas and LCCs. Finally, no expression of either desmocollin-3 or TTF-1 was identified in sarcomatoid carcinomas.

\section{Control cases}

Control squamous carcinomas had a diffuse desmocollin-3 expression in 20 of the 20 cases (Figure 1a-c), more intense in keratinized areas, whereas TTF-1 reactivity was generally absent (a weak positivity was present in two cases). The adenocarcinomas contained TTF-1 in $90 \%$ of cases (18 of the 20 ) and desmocollin-3 was focally expressed in 1 case only (possibly representing a focal area of squamous differentiation, upon revision of the hematoxylin and eosin-stained slides). In all three adenosquamous carcinomas, focal expression of both TTF-1 and desmocollin-3 was observed in different tumor areas and no co-expression of the two markers in the same cell was observed in a case submitted to a double immunohistochemical stain.

\section{Cytological Material}

All cytological samples as well as the corresponding bronchial biopsies and, whenever available, surgical specimens were immunostained for desmocollin-3, p63 and TTF-1. In terms of quality of the staining, no difference in reactivity was observed between the cell blocks of fine-needle aspirates and bronchoalveolar aspirates.

In the 31 cytological samples originally diagnosed as 'non-small-cell lung carcinomas', desmocollin-3 was expressed in 5 cases and TTF-1 in 9 cases, respectively (Figure 2). The remaining 17 cases were negative for both markers. p63 was focally detected in 15 tumors (including all desmocollin-3-reactive cases, but also a fraction of tumors expressing TTF-1). No case had concurrent reactivity for TTF-1 and desmocollin-3. Comparing these results

Table 1 Clinicopathological data and marker expression in 62 resected undifferentiated LCCs of the lung

\begin{tabular}{|c|c|c|c|c|c|c|}
\hline & $\begin{array}{l}\text { Samples } \\
\text { (no. 29) }\end{array}$ & & & $\begin{array}{c}\text { Validation } \\
\text { cohort (no. 33) }\end{array}$ & & \\
\hline \multicolumn{7}{|l|}{ Clinical data } \\
\hline Age (years) & $64(45-80)$ & & & $62(45-83)$ & & \\
\hline Sex (male/female) & $22 / 7$ & & & $29 / 4$ & & \\
\hline Mean tumor size $(\mathrm{cm})$ & 4.1 & & & 4.0 & & \\
\hline Histotype & & DSC3 & $T T F-1$ & & DSC3 & $T T F-1$ \\
\hline Large-cell carcinoma NOS (ICD-O code 8012/3) & $16 / 29$ & $8 / 16^{\mathrm{a}}$ & $8 / 16^{\mathrm{a}}$ & $20 / 33$ & $5 / 20^{\mathrm{a}}$ & $11 / 20^{\mathrm{a}}$ \\
\hline Large-cell carcinoma, clear-cell variant (ICD-O code 8310/3) & $7 / 29$ & $3 / 7^{\mathrm{a}}$ & $4 / 7^{\mathrm{a}}$ & $5 / 33$ & $4 / 5^{\mathrm{a}}$ & $1 / 5^{\mathrm{a}}$ \\
\hline Large-cell carcinoma, basaloid variant (ICD-O code 8123/3) & $3 / 29$ & $3 / 3^{\mathrm{a}}$ & $0 / 3$ & $3 / 33$ & $3 / 3^{\mathrm{a}}$ & $0 / 3^{\mathrm{a}}$ \\
\hline Large-cell carcinoma, lymphoepithelioma-like (ICD-O code 8082/3) & $0 / 29$ & - & - & $1 / 33$ & $1 / 1^{\mathrm{a}}$ & $0 / 1$ \\
\hline Sarcomatoid carcinoma, spindle-cell variant (ICD-O code 8032/3) & $3 / 29$ & $0 / 3$ & $0 / 3$ & $4 / 33$ & $0 / 4$ & $0 / 4$ \\
\hline \multicolumn{7}{|l|}{ Control groups } \\
\hline Squamous carcinoma & 20 & $20 / 20$ & $2 / 20$ & & & \\
\hline Adenocarcinomas & 20 & $1 / 20$ & $18 / 20$ & & & \\
\hline Adenosquamous carcinoma & 3 & $3 / 3$ & $3 / 3$ & & & \\
\hline
\end{tabular}

${ }^{\mathrm{a}}$ Mutually exclusive.

Figure 1 Desmocollin-3 and TTF-1 immunoreactivities in a control case of squamous carcinoma of the lung (a-c), in various subtypes of
large-cell lung carcinomas $(\mathbf{d}-\mathbf{o})$ and in sarcomatoid carcinoma $(\mathbf{p}-\mathbf{r})$. Neoplastic squamous cells are strongly positive for desmocollin-3
at the membrane level $(\mathbf{b})$, whereas TTF-1 is negative in the same case $(\mathbf{c})$. Basaloid variant of large-cell carcinoma $(\mathbf{d})$ also expresses
diffusely desmocollin-3 (e), in the absence of TTF-1 reactivity $(\mathbf{f})$. Clear-cell variant of large-cell carcinoma (g, j) has a variable phenotypic
profile, being some cases expressing desmocollin-3 but not TTF-1 (h, i), whereas others have the reverse profile, with mutually exclusive
reactivity patterns $(\mathbf{k}, \mathbf{l})$. A large-cell carcinoma NOS having a marked lymphoid infiltration $(\mathbf{m})$ expresses TTF-1 only, in the absence of
desmocollin-3 reactivity (n, o). Finally a sarcomatoid carcinoma having predominant spindle-cell features $(\mathbf{p})$ was completely negative
for both markers (q, r). (First column to the left: hematoxylin and eosin, original magnification $\times 200$; middle and right columns:
immunoperoxidase for desmocollin-3 and TTF-1, respectively, original magnification $\times 200$.) 

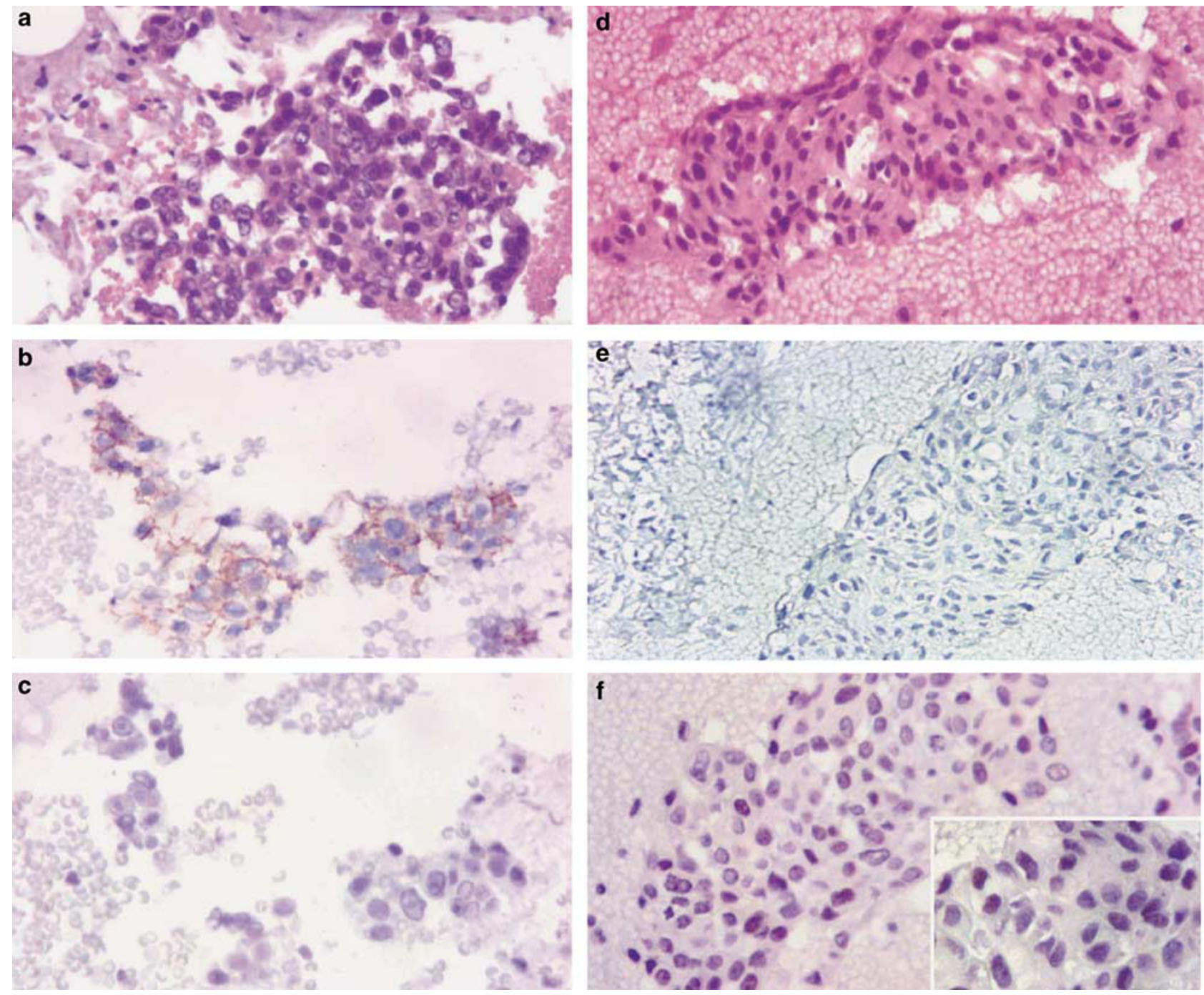

Figure 2 Desmocollin-3 and TTF-1 immunoreactivities in two poorly differentiated lung cancers reported as 'NSCLC' in cytological specimens. In the first case, the bronchoalveolar lavage (a) showed desmocollin-3 reactivity (b), in the absence of TTF-1 (c) in serial sections of the cell block; the case was subsequently diagnosed as poorly differentiated squamous carcinoma in the surgical specimen. The other case (d), which was histologically proven to be a large-cell carcinoma, did not contain any desmocollin-3 reactivity (e), but diffusely expressed TTF-1 (f) in serial sections of the cell block of the transthoracic fine-needle aspiration biopsy. (a, d: hematoxylin and eosin, original magnification $\times 200$; b, $\mathbf{c}, \mathbf{e}, \mathbf{f}$ : immunoperoxidase for desmocollin-3 (b, e) and TTF-1 (c, f), original magnification $\times 200$.)

with the final histopathological diagnosis, desmocollin-3 reactivity was present in all four squamous carcinomas and 1 of the 7 large-cell cancers, whereas TTF- 1 was expressed in 7 of the 20 adenocarcinomas and 2 of the 7 large-cell cancers. The 17 double marker negative tumors were either adenocarcinomas $^{13}$ or LCCs. ${ }^{4}$

Immunohistochemical tests run in parallel on the corresponding histological samples (18 surgical specimens and 13 bronchial biopsies of inoperable patients) had a partially overlapping reactivity. Desmocollin-3 was detected in the same five cases also positive in cytological specimens. TTF-1 was expressed in 21 tumors (including all 9 cases also positive in cytology material), all mutually exclusive with desmocollin-3. The remaining five cases were negative for both markers. p63 immunoreactivity was present in 20 of the 31 cases, including all 5 cases also reactive for desmocollin-3 and a fraction of large cell and adenocarcinomas. Regarding final histopathological diagnosis, desmocollin-3 reactivity was present in all 4 squamous carcinomas and 1 of the 7 large-cell cancers, whereas TTF-1 was expressed in 16 of the 20 adenocarcinomas and 5 of the 7 large-cell cancers. The five double marker negative cases on histological samples included four adenocarcinomas and one LCC.

In summary, TTF-1 expression was concordant in the cytological samples and the corresponding histological material of nine TTF-1-positive cases and four negative tumors (overall agreement of $62 \%$ ). In eight tumors, TTF-1 was focally expressed in the histological sample, only, and missed in the cytological materials. Conversely, desmocollin-3 
Table 2 Phenotypic marker expression in 31 cytological samples originally diagnosed as 'NSCLC not otherwise specified' and in their corresponding biopsy or surgical specimens

\begin{tabular}{|c|c|c|c|c|}
\hline \multirow{2}{*}{ Clinical data } & \multicolumn{2}{|c|}{ Age (years, median) } & \multicolumn{2}{|c|}{64 (range 45-80) } \\
\hline & \multicolumn{2}{|c|}{ Sex (male/female) } & \multicolumn{2}{|l|}{$28 / 3$} \\
\hline \multirow[t]{2}{*}{ Diagnosis } & \multicolumn{2}{|c|}{ Cytological samples } & \multirow{2}{*}{\multicolumn{2}{|c|}{$\begin{array}{l}\text { Histological samples } \\
\text { Squamous carcinoma 4/31 } \\
\text { Adenocarcinoma 20/31 } \\
\text { Large-cell carcinoma } 7 / 31\end{array}$}} \\
\hline & NSCLC : & & & \\
\hline Markers & DSC3 & TTF-1 & DSC3 & TTF-1 \\
\hline Squamous carcinoma & $4 / 4$ & $0 / 4$ & $4 / 4$ & $0 / 4$ \\
\hline Adenocarcinoma & $0 / 20$ & $7 / 20$ & $0 / 20^{\mathrm{a}}$ & $16 / 20^{\mathrm{a}}$ \\
\hline Large-cell carcinoma & $1 / 7$ & $2 / 7$ & $1 / 7^{\mathrm{a}}$ & $5 / 7^{\mathrm{a}}$ \\
\hline Total & $5 / 31^{b}$ & $9 / 31^{c}$ & $5 / 31^{b}$ & $21 / 31^{\mathrm{c}}$ \\
\hline
\end{tabular}

NSCLC, non-small-cell lung carcinoma.

${ }^{\mathrm{a}}$ Four adenocarcinomas and one LCC were double negative.

${ }^{\mathrm{b}}$ Overall agreement for DSC3 in cytology vs histology samples: $100 \%$.

${ }^{\mathrm{C}}$ Overall agreement for TTF-1 in cytology vs histology samples: $62 \%$.

was found in both cytological and histology materials of all five positive cases (Table 2).

\section{Discussion}

In this study, we have demonstrated that desmocollin-3, a marker of normal and well-differentiated neoplastic squamous epithelia, is also focally expressed in a fraction of large-cell lung carcinomas, thus allowing to trace residual signs of squamous differentiation, as opposed to glandular differentiation, in the group of 'anaplastic LCC' of the lung.

The current WHO classification of lung tumors ${ }^{3}$ already restricted the category of anaplastic LCC to well-defined, generally rare variants (clear-cell, basaloid, rhabdoid variants as well as the largecell neuroendocrine carcinoma subtype). To this purpose, those carcinomas having a markedly pleomorphic appearance, including giant cell types, spindle-cell growth or extensive anaplasia, were moved into a separate category of sarcomatoid carcinoma. Theoretically, this proposal is highly meaningful, but in the daily practice there are cases extremely undifferentiated or having combined features, so that a reliable subclassification is sometimes difficult, especially in small biopsy samples and the category 'LCC' may sometimes be selected by exclusion.

The correct identification of squamous or glandular types is nowadays becoming clinically relevant for tailoring the appropriate systemic treatment and it is not clearly well established how undifferentiated cancers respond to such treatments, due to the heterogeneity of this category. A more accurate profiling of undifferentiated carcinomas might be envisaged using a panel of markers associated to either squamous or glandular lineages. TTF-1 is a nuclear transcription factor that regulates growth and differentiation in thyroid and lung tissues. ${ }^{19}$ Although the current literature data indicate that TTF-1 is highly expressed in most types of lung adenocarcinomas, it has also been reported in a fraction of squamous carcinomas. ${ }^{8-10}$ In our control cases, only two squamous carcinomas $(10 \%)$ were positive for TTF-1, which was conversely expressed in a percentage of LCCs, as expected from the literature. Concerning markers usually associated with squamous-cell carcinoma differentiation, we did not find p63 to be specific enough of the squamous-cell population, and indeed several adenocarcinomas were also positive, as also recently reported. ${ }^{11}$

Conversely, desmocollin-3 seemed specific for squamous histotype and indeed also a percentage of undifferentiated LCCs had a more or less extensive reactivity. Interestingly, this was mutually exclusive with TTF-1, thus allowing to associate a fraction of undifferentiated non-small-cell cancers to either squamous or glandular lineages. In this line, clear-cell carcinoma variants of LCC may contain glycogen or mucins. In the absence of squamous or glandular differentiation, the tumor cannot be classified as clear-cell variant of either squamous or adenocarcinoma, although it probably represents a high-grade counterpart of one of the two types as already suggested more than 25 years ago by Katzenstein et al. ${ }^{20}$ These authors proposed that most, if not all, clear-cell carcinomas of the lung are a nonentity, but rather represent clear-cell changes within common types of cancers, either squamous carcinoma or adenocarcinoma. In our series, approximately half cases expressed TTF-1 and the other half contained desmocollin-3-reactive cells, thus indicating a relationship with one of the two main lineages of non-small-cell lung cancer. The same distinction might be obtained with PAS and PAS after diastase staining, but these histochemical stains appeared more difficult to interpret, especially in small biopsy samples, also due to background staining. For example, the differential diagnosis of LCC from solid variant with mucin production of adenocarcinoma is based on the recognition of at least five mucin droplets in two high-power fields. ${ }^{3}$ This feature can be easily missed in extensively necrotic tumors and/or in small biopsy samples. Lineage markers may provide a better definition of the tumor cell features/origin, and residual expression of either desmocollin-3 for squamous phenotypes or of surfactant (unfortunately not produced by all subtypes and variants) or, better, TTF-1 might be a more accurate alternative. Within the recently introduced category of sarcomatoid carcinoma (accounting for no more than $1.3 \%$ of pulmonary malignancies), ${ }^{3}$ no TTF-1 or desmocollin-3 immunoreactivity was found in six cases analyzed, probably as a result of extreme dedifferentiation.

Such phenotypic profiling is of potential interest to better select the appropriate chemotherapy regimen also for patients with a generic diagnosis of undifferentiated carcinoma, according to the observed differential efficacy of individual drugs or 
combinations with regard to individual histotypes. The observed better response rate and survival in adenocarcinoma compared to squamous-cell carcinoma recently reported with cisplatin and pemetrexed, as opposed to a better response and survival of squamous-cell carcinoma to cisplatin and gemcitabine,${ }^{4}$ and the proven benefit of adding bevacizumab to carboplatin and paclitaxel to improve the outcome of non-squamous cancer patients ${ }^{5,6}$ indicate the clinical need to fine-tune the tailored approach in undifferentiated lung carcinomas. Owing to their rarity and also the recent subclassification (large-cell variants and sarcomatoid carcinoma variants), a molecular or immunochemical profiling would be very useful, in the absence of specific data about their clinical response to chemotherapy, to understand if at least part of LCCs is more closely related to adenocarcinoma or squamous-cell carcinoma.

The enzyme thymidylate synthase was differently expressed in adenocarcinoma and squamous-cell carcinoma and preliminary data in LCC indicate expression levels more closely related to those detected in squamous-cell carcinoma. ${ }^{21}$ Similarly, the DNA repair gene ERCC1 was more highly expressed in squamous carcinomas than adenocarcinomas, but LCCs were not specifically analyzed, ${ }^{22}$ although in another study expression levels of this gene were not correlated with specific histotypes. ${ }^{23}$

Finally, in this study we demonstrated that the immunoprofile can be tentatively defined in at least part of cytological or biopsy samples obtained during the diagnostic work-up of a lung tumor mass. Owing to the generally limited amount of viable cells, the generic diagnosis of non-small-cell lung carcinoma has been largely used in a variable percentage of these specimens. ${ }^{24}$ Although not preventing the possibility of combined squamous and adenocarcinomas, the immunostaining for desmocollin-3 and at least TTF-1 may help to identify focal areas of either residual differentiation or at least a signature of the predominant lineage of origin. This could provide further support to the choice of the most appropriate chemotherapy especially for inoperable patients, whose exact tumor type definition might remain pending because of scant cellularity and/or extreme undifferentiation. The effects of such further subtyping obviously need to be analyzed in relationship with the response to targeted treatments designed for specific histological groups.

In conclusion, we have shown that the squamouscell marker desmocollin-3 is expressed in a fraction of LCCs of the lung and it is mutually exclusive with TTF-1 (expressed by control adenocarcinomas and part of LCCs). Undifferentiated lung cancers are often hard to subtype, especially on biopsy or cytological material, and a panel of markers including desmocollin-3 and TTF-1 may be a useful adjunct to morphology. Whether a more or less extensive expression of squamous and glandular markers in undifferentiated lung cancers may affect the response to selectively active therapeutic regimens is unclear. Specific trials should be designed on such tumor subgroup, taking into consideration the relative extent of the individual components, which possibly reflect the different genetic signatures already identified by gene array data in the various histotypes of lung cancer.

\section{Acknowledgements}

This study was supported by grants from the Italian Ministry of University (Rome, ex 60\% to MP and GVS) and the Regione Piemonte (Turin, Ricerca Sanitaria Finalizzata, to MP and GVS). VM is recipient of a scholarship from the San Luigi Hospital Pulmonary Oncology Unit.

\section{Conflict of interest}

All the Authors have no conflict of interest to declare.

\section{References}

1 Hirsch FR, Spreafico A, Novello S, et al. The prognostic and predictive role of histology in advanced non-small cell lung cancer: a literature review. J Thorac Oncol 2008;3:1468-1481.

2 Travis WD, Colby TV, Corrin B. Histological Typing of Lung and Pleural Tumours. WHO International Histological Classification of Tumours, 3rd edn. Springer: Berlin, 1999.

3 Travis WD, Brambilla E, Müller-Hermelink HK, et al. World Health Organisation Classification of Tumors. Pathology and Genetics of Tumors of the Lung, Pleura, Thymus and Heart, 4th edn. IARC Press: Lyon, 2004.

4 Scagliotti GV, Parikh P, von Pawel J, et al. Phase III study comparing cisplatin plus gemcitabine with cisplatin plus pemetrexed in chemotherapy-naive patients with advanced-stage non-small-cell lung cancer. J Clin Oncol 2008;26:3543-3551.

5 Johnson DH, Fehrenbacher L, Novotny WF, et al. Randomized phase II trial comparing bevacizumab plus carboplatin and paclitaxel with carboplatin and paclitaxel alone in previously untreated locally advanced or metastatic non-small-cell lung cancer. J Clin Oncol 2004;22:2184-2191.

6 Aita M, Fasola G, Defferrari C, et al. Targeting the VEGF pathway: antiangiogenic strategies in the treatment of non-small cell lung cancer. Crit Rev Oncol Hematol 2008;68:183-196.

7 Downey P, Cummins R, Moran M, et al. If it's not CK5/6 positive, TTF-1 negative it's not a squamous cell carcinoma of lung. APMIS 2008;116:526-529.

8 Fabbro D, Di Loreto C, Stamerra O, et al. TTF-1 gene expression in human lung tumours. Eur J Cancer 1996;32A:512-517.

9 Tan D, Li Q, Deeb G, et al. Thyroid transcription factor-1 expression prevalence and its clinical implications in non-small cell lung cancer: a high-throughput 
tissue microarray and immunohistochemistry study. Hum Pathol 2003;34:597-604.

10 Pelosi G, Fraggetta F, Pasini F, et al. Immunoreactivity for thyroid transcription factor-1 in stage I non-small cell carcinomas of the lung. Am J Surg Pathol 2001;25: 363-372.

11 Nonaka D, Chiriboga L. Basal cell differentiation in lung adenocarcinoma. [abstract]. Mod Pathol 2008; 21S:348A.

12 Rossi G, Marchioni A, Milani M, et al. TTF-1, cytokeratin 7, 34betaE12, and CD56/NCAM immunostaining in the subclassification of large cell carcinomas of the lung. Am J Clin Pathol 2004;122:884-893.

13 Chen HY, Yu SL, Chen CH, et al. A five-gene signature and clinical outcome in non-small-cell lung cancer. N Engl J Med 2007;356:11-20.

14 Hayes DN, Monti S, Parmigiani G, et al. Gene expression profiling reveals reproducible human lung adenocarcinoma subtypes in multiple independent patient cohorts. J Clin Oncol 2006;24:5079-5090.

15 Motoi N, Szoke J, Riely GJ, et al. Lung adenocarcinoma: modification of the 2004 WHO mixed subtype to include the major histologic subtype suggests correlations between papillary and micropapillary adenocarcinoma subtypes, EGFR mutations and gene expression analysis. Am J Surg Pathol 2008;32:810-827.

16 Angulo B, Suarez-Gauthier A, Lopez-Rios F, et al. Expression signatures in lung cancer reveal a profile for EGFR-mutant tumours and identify selective PIK3CA overexpression by gene amplification. J Pathol 2008;214:347-356.
17 Fukuoka J, Dracheva T, Shih JH, et al. Desmoglein 3 as a prognostic factor in lung cancer. Hum Pathol 2007;38:276-283.

18 Legan PK, Yue KK, Chidgey MA, et al. The bovine desmocollin family: a new gene and expression patterns reflecting epithelial cell proliferation and differentiation. J Cell Biol 1994;126:507-518.

19 Lazzaro D, Price M, de Felice M, et al. The transcription factor TTF-1 is expressed at the onset of thyroid and lung morphogenesis and in restricted regions of the foetal brain. Development 1991;113:1093-1104.

20 Katzenstein AL, Prioleau PG, Askin FB. The histologic spectrum and significance of clear-cell change in lung carcinoma. Cancer 1980;45:943-947.

21 Ceppi P, Volante M, Saviozzi S, et al. Squamous cell carcinoma of the lung compared with other histotypes shows higher messenger RNA and protein levels for thymidylate synthase. Cancer 2006;107:1589-1596.

22 Olaussen KA, Dunant A, Fouret P, et al. DNA repair by ERCC1 in non-small-cell lung cancer and cisplatinbased adjuvant chemotherapy. N Engl J Med 2006;355: 983-991.

23 Ceppi P, Volante M, Novello S, et al. ERCC1 and RRM1 gene expressions but not EGFR are predictive of shorter survival in advanced non-small-cell lung cancer treated with cisplatin and gemcitabine. Ann Oncol 2006;17:1818-1825.

24 Edwards SL, Roberts C, McKean ME, et al. Preoperative histological classification of primary lung cancer: accuracy of diagnosis use of the non-small cell category. J Clin Pathol 2000;53:537-540. 